\title{
HAP - A Heterogeneous Ad hoc Protocol
}

\author{
Arta Doci \\ Colorado School of Mines \\ Golden, Colorado \\ adoci@mines.edu
}

\author{
Leonard Barolli \\ Fukuoka Institute of Technology \\ Fukuoka, Japan \\ barolli@ fit.ac.jp
}

\author{
Fatos Xhafa \\ Technical University of Catalonia \\ Barcelona, Spain \\ fatos@1si.upc.edu
}

\begin{abstract}
Ad hoc wireless networks are becoming an important infrastructure for developing networking applications due to their decentralized nature, improved scalability as compared to wireless managed networks, minimal configuration and fast deployment. However, such networks show several limitations regarding their capacity and are, in general, impractical for stand alone commercial applications; in fact, such networks are mostly used for emergency applications. However, the features of ad hoc networks can be useful to extend the connectivity of wireless networks, while taking into account node mobility. In this paper we propose a Heterogeneous Ad hoc Protocol (HAP), which aims to extend the connectivity of a wireless networks. HAP is a cross layer protocol that operates on the link, MAC, and network layers, which takes into account the impact of the mobility on each of these layers. The objective of HAP is thus to overcome limitations of existing protocols, especially regarding mobility. HAP can be useful for developing applications in pedestrian and community networks.
\end{abstract}

\section{Introduction}

Ad hoc networks have been proven useful in military and emergency applications, however they have been unable to take off as a business model for commercial applications. The main reasons for being unable to begone as commercial applications are the constraints of bandwidth, energy, and security. In addition, the scalability of these applications offers a trade off on protocol performance.

Despite their limitations $[1,2,3]$, their decentralized nature, improved scalability as compared to wireless managed networks, minimal configuration and fast deployment makes ad hoc networks an important network infrastructure. In this paper we show that ad hoc networks can be used to extend the connectivity between two wireless networks and can be proven very useful in the campus and community networks. Recent studies on extraction of the mobility properties in the wireless networks for pedestrian [4] and vehicular [5] scenarios have revealed important findings. One such finding is that pedestrian and vehicular mobility models have many similarities in common. First, they show that the wireless nodes tend to cluster around popular locations (hotspots). Second, the selection of the source and destination pairs, as well as the movement from source toward the destinations is not at random, but rather it is activity based.

For example, in Fig. 1, we display four hotspots on the Ohio State University student campus map, namely, $\mathbf{R}$ (Recreational/Athletics), $\mathbf{H}$ (Housing), $\mathbf{W}$ (Wireless) and $\mathbf{L}$ (Library).

In general, due to the distributions of hotspots, there might be connectivity gaps between the hotspots, which could negatively impact on network connectivity and performance. This paper is motivated by the need to avoid connectivity gaps in wireless networks. To this end, we address the use ad hoc networks of mobile wireless devices to extend the connectivity among hotspots. To achieve this, we propose a Heterogeneous Ad hoc Protocol (HAP), which aims to extend the connectivity of a wireless networks. HAP is a cross layer protocol that operates on the link, MAC, and network layers, which takes into account the impact of the mobility on each of these layers. The objective of HAP is thus to overcome limitations of existing protocols, especially regarding mobility.

HAP, first decides on the fly the number of hotspots that it is going to operate into. Next, within a hotspot, HAP considers the nodes to be stationary and connected using the wireless network with infrastructure. Therefore, the proto- 
col, when operating within the cluster, considers the constraints of battery and bandwidth as negligible.

In addition, within the cluster the protocol adopts the table driven approach; therefore, each node within the cluster has full knowledge of the cluster topology. Second, HAP assigns the nodes that are in the radius of the cluster $( \pm \delta$, where $\delta$ is $5 \% \times$ Radius), as the edge nodes. The edge nodes periodically sent hello messages outside of the cluster, thus extend the connectivity to the mobile nodes outside of the cluster. The edge nodes, also can be thought of as gateways for the nodes within and outside the cluster. Third, nodes outside the cluster, since they are mobile, adopt the cross layer AODV protocol [6]. The cross layer approach takes into account mobility into the AODV design.

In this paper, the cross layer approach focuses on the following three layers:

1. Link Layer: the frequency that the links characteristics change with time;

2. MAC Layer: the validity of the channel state and interferences;

3. Network Layer: the performance of the routing protocols due to rapid changes in topology.

The contributions of this paper are two fold. The first contribution is the design of HAP, a Heterogeneous Ad hoc Protocol. The second contribution is that it applies the protocol into two scenarios, namely student campus and community networks.

The rest of the paper is organized as follows. In Section 2 a brief review of most used ad hoc protocols is provided. The main properties of the proposed HAP protocol are discussed in Section 3 and its design is presented in Section 4. We end the paper in Section 5 with some conclusions.

\section{Overview of Ad Hoc Protocols}

In this section we provide a review of the most used ad hoc protocols and justify our design choices for HAP. The main groups of ad hoc protocols can be divided into reactive/on demand, proactive/table driven, hybrid of reactive and proactive, hierarchical/clustered, and geographical based routing. First, we briefly describe a representative from each group and in the end we justify the choices for HAP.

\subsection{Reactive or On Demand Ad hoc Protocols}

The reactive protocols will ask for a route and discover it only when needed. The most used protocols in this group are Ad hoc On-Demand Distance Vector $(A O D V)$ [6] and Dynamic Source Routing ( $D S R$ ) [7]. Both these protocols are classified as on-demand protocols, due to the fact that a route is discovered only when a packet is needed to be delivered. When a source needs to send data to a destination, it issues a route request $(R R E Q)$, until the destination is found. After the destination is found, a route reply $(R R E P)$ is send back to the source. If errors occur due to link failures, a route error $(R R E R)$ will be sent back to the source. In addition, two other similarities between the two protocols are the requirement of bidirectional links (due to the need of $R R E P$ to propagate back) and the use of flooding.

\subsection{Proactive or Table Driven Ad hoc Protocols}

The table driven protocols keep updated information for each node in the network. Each node maintains one or more tables to store the routing information, as well as, respond to the topology changes by propagating the changes to all the nodes in the network.

The most used table driven protocol is DestinationSequenced Distance-Vector Routing ( $D S D V$ ) protocol [8]. In $D S D V$ each mobile node in the network keeps a routing table with all the possible destinations and the hops to each destination. Each row in the table is sequenced by a number that helps to distinguish between the stale routes and new ones. Routing tables are propagated periodically.

\subsection{Hierarchical or Clustered Ad hoc Protocols}

In these group the mobile nodes are assigned into clusters and a cluster head is elected using a distributed algorithm. All nodes in the communication range of the cluster head belong to its cluster. A node that is in the communication range of two or more cluster heads is called a gateway node.

One example is the Cluster-Head Gateway Switch Routing Protocol $(C G S R)$ [9]. A clusterhead is able to control a group of ad hoc nodes, by broadcasting within the cluster, forwarding messages, and dynamically providing the channel scheduling.

\subsection{Geographical based Ad hoc Protocols}

These protocols use location information that can be obtained using the global positioning system (GPS). One representative is Location-Aided Routing ( $L A R$ ) protocol [10], which uses location information to narrow the search space results in fewer route discovery messages.

\section{HAP's Main Properties}

The main issue across all the above protocols is that none of them is designed to take into account the mobility in the 


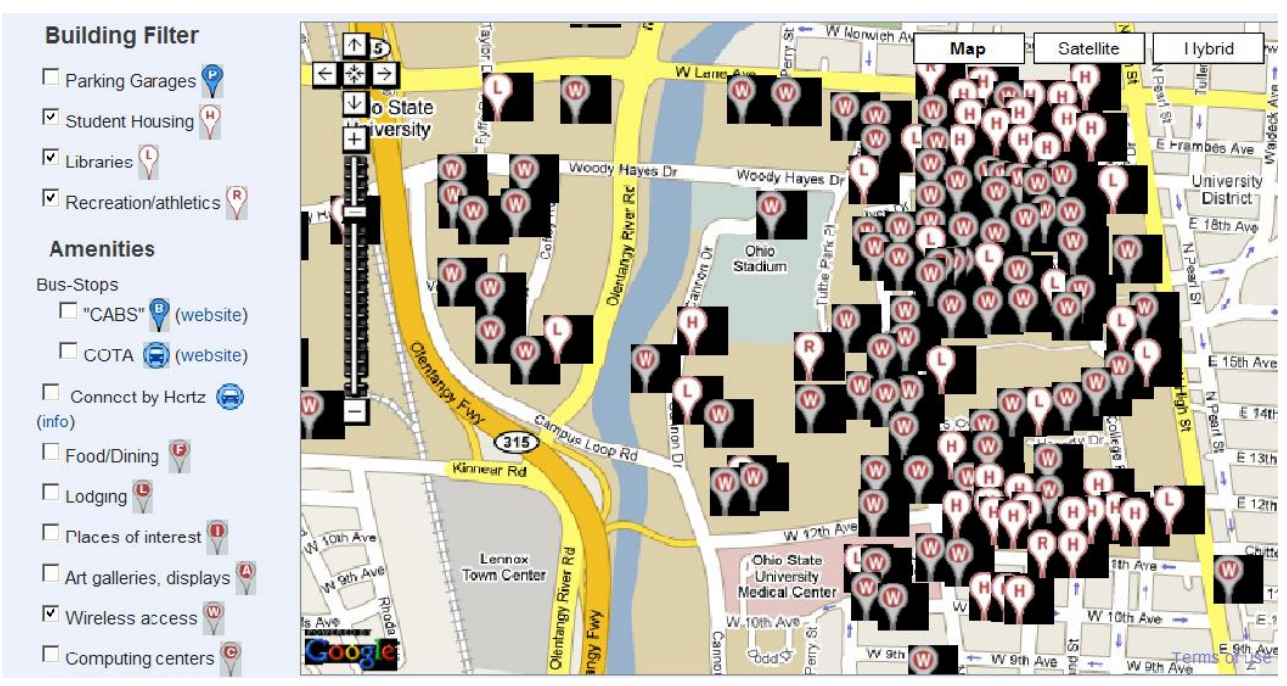

Figure 1. Popular locations on a campus map.

protocol design space. The design of HAP protocol is thus motivated by the need to make mobility part of the protocol design.

First, real mobility models suggest that wireless nodes are clustered around popular locations. In fact, ClusterHead Gateway Switch Routing Protocol (CGSR) tries to address this constraint, however the main drawback is that it does so unrealistically. The nodes are assigned into clusters, but without knowledge of the hotspots or the nodes relative positions to the hotspots. HAP addresses this issue by automatically clustering the nodes based on their location, hotspot information, and relative position of the node to the hotspot.

Second, the main bottleneck in ad hoc networks is the low protocol performance due to mobility of the wireless nodes. While, mobility impacts the entire protocol stack of a communication network with mobile nodes; yet, mobility has not been made part of the protocol design.

Third, nodes in the network do not move at random, but rather follow popular locations and directions. Therefore, to improve the protocol performance we broadcast toward the nodes directions and avoid flooding the network in the wrong direction.

HAP proposes a cross layer design for the mobile nodes. One of the main advantages of the cross layer design is that it enables improved overall network performance and increased network lifetime $[11,12,13,14]$.

\section{HAP Design}

The design of HAP protocol is done as follows. The first step is to ensure that each node knows it's geographical location and is able to report it's $(x, y)$ coordinates. As shown in Figure 1, the number of hotspots can be large, thus HAP assesses the right number of hotspots on a threshold value for the number of data points on each hotspot.

The second step looks into the nodes within the cluster, since they belong to the same hotspot and are considered connected. These nodes are called stationary nodes. We apply the modified $D S D V$ protocol within the cluster. The first modification when within the cluster is that the nodes are separated in two types, namely edge and cluster nodes. The cluster nodes maintain a routing table that stores the destinations, number of the hops and the sequential numbers. The second modification is that within the cluster, each edge node maintains two routing tables, instead of one. One of the tables is the same as the clustering nodes, while the other one stores the information about the nodes outside of the clusters.

The third step, considers the nodes between the clusters. These nodes are considered as mobile and they take mobility into the protocol design, by proposing a cross layer architecture. In this paper, we focus on the following three layers (see Fig. 2) only:

1. Link Layer: Frequency that the links characteristics change with time;

2. MAC Layer: Validity of the channel state and interferences;

3. Network Performance of the routing protocols due to rapid changes in topology.

\subsection{Mobility parameters in protocol design}

In this section we identify the mobility parameters to be included in the design ad hoc protocols. 


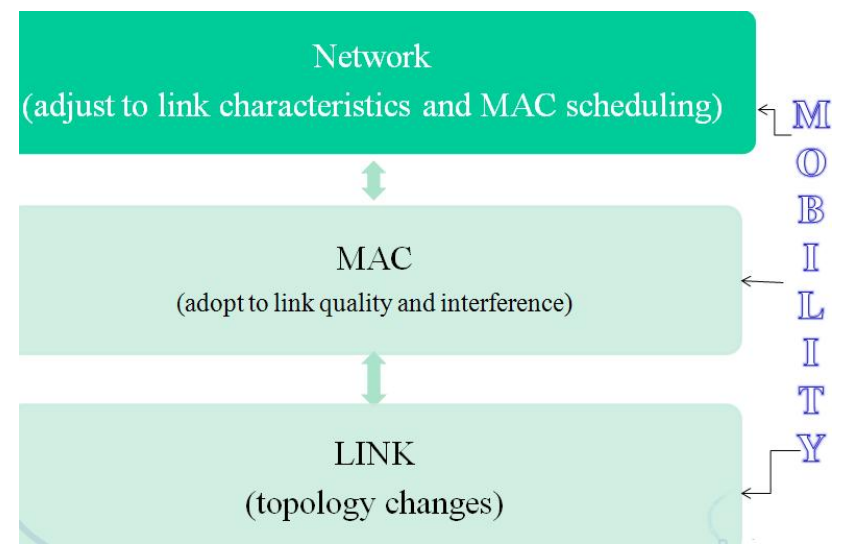

Figure 2. Cross layer design for mobile nodes.

Speed Distribution. Speed will impact all the three layers showed in Fig. 2. Therefore, we take it into the protocol design and fine tune the parameters for each scenario at hand. For example, in the pedestrian case the speed is withdrawn from a lognormal distribution [4]. If the mobile nodes are not very sparse, the protocol performance of the delivery ratio stayed at about $90 \%$.

In the case of the community network, where we consider as mobile nodes the vehicular inter city traffic, the speed distribution [15] of mixed traffic is best modeled by bimodal distribution. The study reveals that the speed distribution in one of the most condensed traffic areas is bimodal with the mean to be 12.5 on the first peak and 37.5 on the second peak.

Direction of Movement. Wireless devices are carried by humans, thus the human movements would be the best approximation to the mobility patterns of the mobile nodes. Humans do not move at random, but rather based on activities. For instance, data collected on student campuses (e.g. Dartmouth Campus) and community networks (e.g. Lexington area [16]) supports that mobile nodes move based on activities (the findings are summarized in Fig. 3).

Dynamic Membership Dynamic membership of wireless nodes is another important feature. In Fig. 4 we can see that $50 \%$ of the trips were on length of $[0,9]$ minutes, $30 \%$ of the trips were on length of $[10,19]$ minutes, $10 \%$ of the trips were on length of $[20,29]$ minutes, $4 \%$ of the trips were on length of $[30,39]$ minutes, $2 \%$ of the trips were on length of $[40,49]$ minutes, and $4 \%$ of the trips were on length of $[50,+]$ minutes.

This demonstrates that wireless nodes stay in the network only for a certain duration of time, therefore their dynamic membership should be taken into account when de-

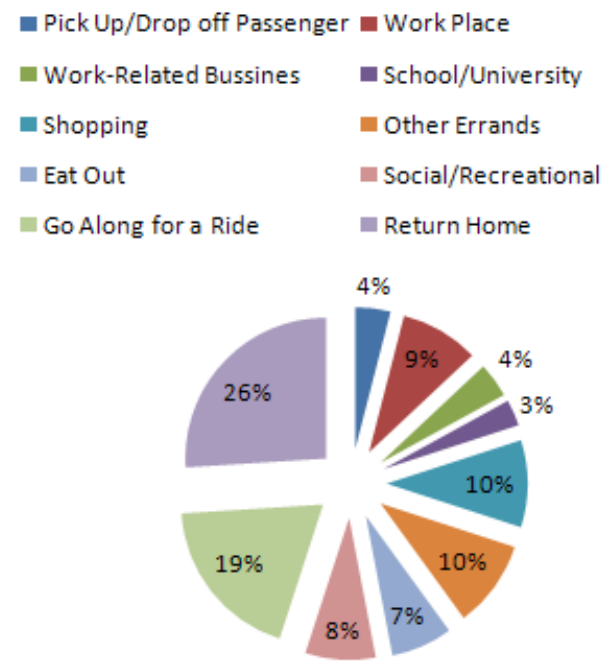

Figure 3. Destinations of movements (in $\%$ of trips).

signing ad hoc protocols.

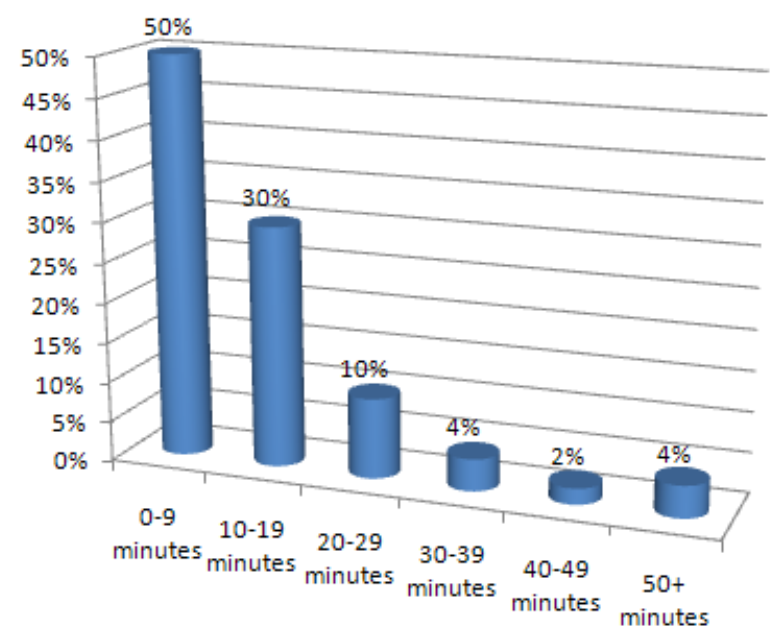

Figure 4. Time duration of trips.

\subsection{Assessing the right number of clusters}

We are considering a set of wireless devices and assume that each device is equipped with a GPS, thus can report it's $(X, Y)$ coordinates. Real mobility models described in the previous sections revealed that wireless devices are clustered around popular locations. Therefore, determining the number of the popular locations that each ad hoc protocol should operate into automatically and assigning each wireless device to the appropriate popular location is the focus 
of this section. While the number of hotspots in a small area on a campus settings can be large, we have set as the threshold for the elements in a cluster to be greater or equal to 50 elements and less or equal to 100 .

In this paper we use the hierarchical clustering to investigate grouping in a data set, simultaneously over a variety of scales, by creating a cluster tree. The tree is not a single set of clusters, but rather a multilevel hierarchy, where clusters at one level are joined as clusters at the next higher level. This generally allows a user to decide what level, scale or complexity of clustering is most appropriate in a particular application.

Determining the exact number of clusters is considered a hard problem. In this paper, we examine the use of a criterion based on the Rissanen's Minimum Description Length [17] Principle to evaluate the results of the hierarchical clustering procedure, in particular to select the appropriate number of clusters.

Hierarchical Clustering (see Alg. 1) takes as input the $n \times n$ distance matrix and $d$ pairwise distances between points. The algorithm is simple and starts with $n$ clusters, one for each data point and constructs a graph $T$ by assigning one vertex to each cluster. Then, it finds the two closest clusters $C 1$ and $C 2$ and merges them into new cluster $\mathrm{C}$ with $|C 1|+|C 2|$ elements. It computes the distance from $C$ to all other clusters and adds a new vertex $C$ to $T$ that connects the to vertices $C 1$ and $C 2$. In addition, it removes the rows and columns of $d$ corresponding to $C 1$ and $C 2$ and adds a row and column to $d$ corresponding to the new cluster $C$.

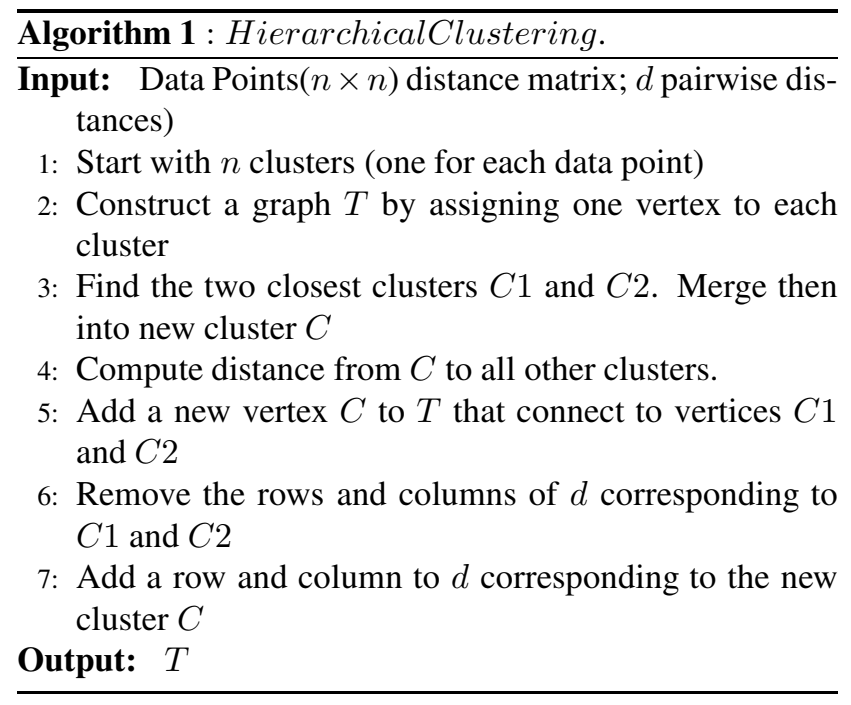

Once the tree is computed, we use it to assess the right number of clusters in the data. In other words we are interested to know where to cut the tree to obtain the right number of clusters in the data. To this end, we use the $M D L$ principle as a criterion for determining the appropriate num- ber of clusters [18], since the method does not depend on the sampling theory.

At each level of a tree with $p$ groups there corresponds a Gaussian model with independent variables. The $M D L$ criterion for the Gaussian model, can be written as follows:

$M D L=\frac{n}{2} \ln \left(\hat{\sigma^{2}}\right)-\ln \Gamma\left(\frac{n-p}{2}\right)+\frac{p}{2} \ln \left(\frac{n R_{0}^{2}}{2 \pi}\right)+\frac{n-p}{2} \ln \left(\frac{n}{2}\right)$.

As an example, we withdraw two 100 random normal data points (20) and plot them in Fig. 5. The results of the tree of the hierarchical clustering for this data set is shown in Fig. 6. We applied the figures of merit from the $M D L$ criterion by [18] and computed the corresponding $M D L$ criterion and select as the "best" hierarchical clustering model the one that yields the lowest $M D L$ value. In this case, the lowest $M D L$ value was reached at two clusters, which corresponds to the true number of clusters.

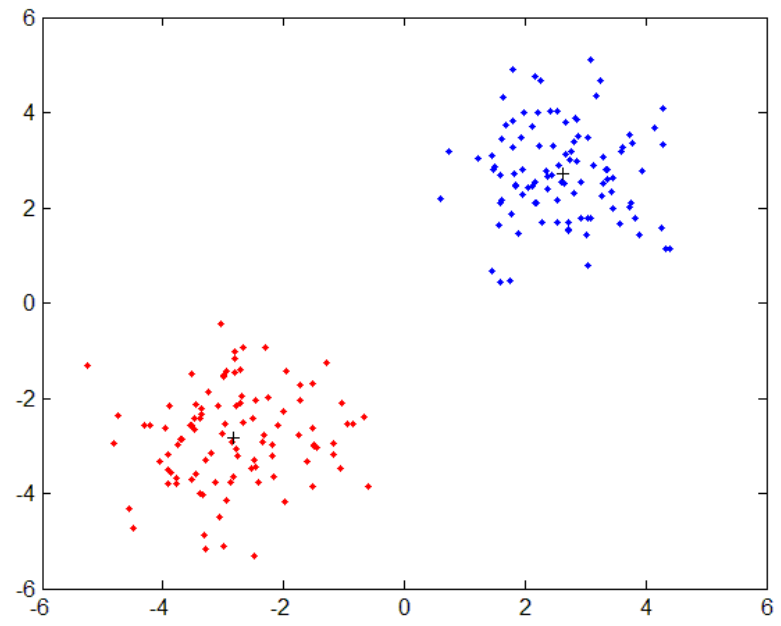

\section{Figure 5. Two gaussian clusters with 100 data points.}

\section{Conclusions}

In this paper we have proposed a Heterogeneous Ad hoc Protocol (HAP) aiming to extend the connectivity of a wireless networks and avoid connectivity gaps. HAP is a cross layer protocol that operates on the link, MAC, and network layers, which takes into account the impact of the mobility on each of these layers. The objective of HAP is thus to overcome limitations of existing protocols, especially regarding mobility. We are in the process of implementing and evaluating HAP in the NS 2 simulator. In addition, we plan to use HAP in a real world implementation to validate the protocol and show its applicability for a pedestrian scenario.

\section{References}

[1] A. Agarwal and P. R. Kumar. "Capacity Bounds for Ad Hoc and Hybrid Wireless Networks", SIGCOMM Comput. Commun. Rev., Vol. 34, No. 3, pp. 71-81, 2004. 


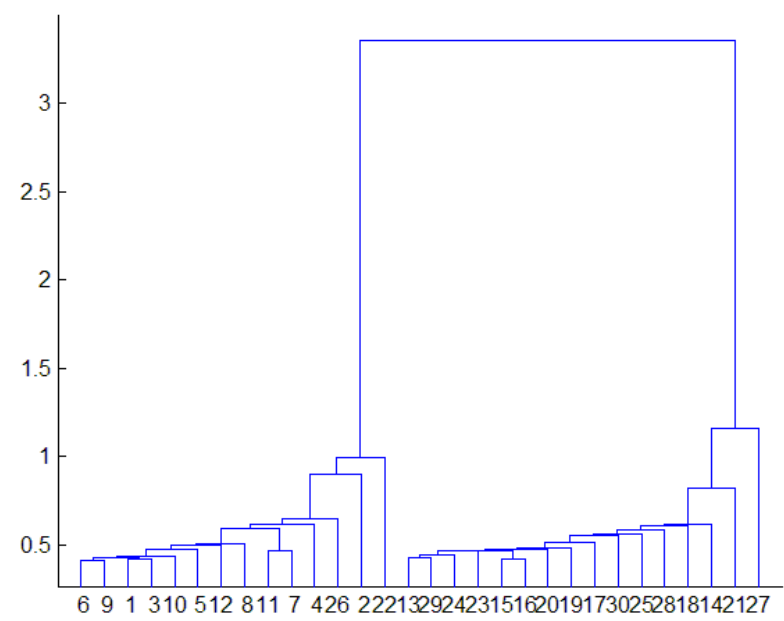

Figure 6. Dendrogram of the hierarchical clustering.

[2] P. Gupta and P. R. Kumar. "Capacity of Wireless Networks", IEEE Transactions on Information Theory, Vol. 46, No. 2, pp. 388-404, 2000.

[3] J. Li, C. Blake, D. S. De Couto, H.I. Lee, and R. Morris. "Capacity of Ad Hoc Wireless Networks", In Proc. of the 7th Annual ACM/IEEE International Conference on Mobile Computing and Networking (MobiCom-2001), pp. 61-69, 2001.

[4] C. Walsh, A. Doci, and T. Camp, "A call to Arms: It's time for Real Mobility Models", SIGMOBILE Mob. Comput. Commun. Rev., Vol. 12, No. 1, pp. 34-36, 2008.

[5] A. Doci, L. Barolli, and F. Xhafa, "Mixmobgen - a realistic mixed traffic mobility generator for ad hoc network simulations", In Proc. of International Conference on Complex, Intelligent, and Software Intensive Systems (CISIS-2009), pp. 335-342, 2009.

[6] C. Perkins and E. Royer, "Ad-hoc On-demand Distance Vector Routing", In Proc. of Mobile Computing Systems and Applications, pp. 90-100, 1999.

[7] D. B. Johnson and D. A. Maltz, "Dynamic Source Routing in Ad Hoc Wireless Networks", Chapter in Mobile Comput- ing, T. Imielinski and H. Korth (Editors), Kluwer Academic Publishers, 1996.

[8] C. Perkins and P. Bhagwat, "Highly Dynamic Destinationsequenced Distance-vector Routing (DSDV) for Mobile Computers", Comp. Commun. Rev., pp. 234-244, 1994.

[9] C. Chiang, "Routing in Clustered Multihop, Mobile Wireless Networks with Fading Channel", In Proc. IEEE SICON'97, pp. 197-211, 1997.

[10] Y. B. Ko and N. H. Vaidya, "Location-Aided Routing (LAR) in Mobile Ad Hoc Networks", Wireless Networks, Vol. 6, No. 4, pp. 307-321, 2000.

[11] Y. Fang and B. McDonald, "Dynamic Codeword Routing (DCR): a Cross-Layer Approach for Performance Enhancement of General Multi-hop Wireless Routing", In Proc. of IEEE SECON, pp. 255-263, 2004.

[12] L. V. Hoesel, T. Nieberg, J. Wu, and P. J. Havinga, "Prolonging the Lifetime of Wireless Sensor Networks by CrossLayer Interaction", IEEE Wireless Communications, Vol. 11, No. 6, pp. 78-86, 2004.

[13] M. C. Vuran, V. B. Gungor, and O. B. Akan, "On the Interdependency of Congestion and Contention in Wireless Sensor Networks", In Proc. of SENMETRICS, pp. 136-147, 2005.

[14] W. Yuan, K. Nahrstedt, S. Adve, D. Jones, and R. Kravets, "GRACE-1: Cross-Layer Adaptation for Multimedia Quality and Battery Energy", IEEE Transactions on Mobile Computing, Vol. 5, No. 7, pp. 799-815, 2006.

[15] P. Dey, S. Chandra, and S. Gangopadhaya, "Speed Distribution Curves under Mixed Traffic Conditions", Journal of transportation engineering, Vol. 132, No. 6, pp. 475-481, 2006.

[16] "Lexington Area Travel Data Collection Test, final report: Global Positioning Systems for Personal Travel Surveys", Battelle Memorial Institute, Available on line at http://www.fhwa.dot.gov/ohim/lextrav.pdf, 1997.

[17] J. Rissanen, "Modeling by Shortest Data Description", Automatica, Vol. 14, pp. 465-471, 1978.

[18] P. G. Bryant, "On the Minimum Description Length (MDL) for Hierarchical Classifications," In Proc. of Data Science, Classification, and Related Methods, pp. 182-187, 1997. 